\title{
Modeling of a compliant joint in a Magnetic Levitation System for an endoscopic camera
}

\author{
M. Simi ${ }^{1}$, N. Tolou ${ }^{2}$, P. Valdastri ${ }^{3}$, J. L. Herder ${ }^{2}$, A. Menciassi ${ }^{1}$, and P. Dario ${ }^{1}$ \\ ${ }^{1}$ The BioRobotics Institute, Scuola Superiore Sant'Anna, Pisa, Italy \\ ${ }^{2}$ Department of Biomechanical Engineering, Faculty of Mechanical, Maritime and Materials Engineering, \\ Delft University of Technology, Delft, The Netherlands \\ ${ }^{3}$ STORM Lab, Mechanical Engineering Department, Vanderbilt University, Nashville, TN, USA
}

Correspondence to: M. Simi (m.simi@sssup.it)

Received: 10 March 2011 - Revised: 6 July 2011 - Accepted: 15 December 2011 - Published: 18 January 2012

\begin{abstract}
A novel compliant Magnetic Levitation System (MLS) for a wired miniature surgical camera robot was designed, modeled and fabricated. The robot is composed of two main parts, head and tail, linked by a compliant beam. The tail module embeds two magnets for anchoring and manual rough translation. The head module incorporates two motorized donut-shaped magnets and a miniaturized vision system at the tip. The compliant MLS can exploit the static external magnetic field to induce a smooth bending of the robotic head $\left(0-80^{\circ}\right)$, guaranteeing a wide span tilt motion of the point of view. A nonlinear mathematical model for compliant beam was developed and solved analytically in order to describe and predict the trajectory behaviour of the system for different structural parameters. The entire device is $95 \mathrm{~mm}$ long and $12.7 \mathrm{~mm}$ in diameter. Use of such a robot in single port or standard multiport laparoscopy could enable a reduction of the number or size of ancillary trocars, or increase the number of working devices that can be deployed, thus paving the way for multiple view point laparoscopy.
\end{abstract}

\section{Introduction}

Minimally invasive surgery reduces pain in the patient and facilitates postoperative recovery by using multiple small abdominal incisions where different instruments are inserted. In the continuous quest to limit access trauma, Single Port Laparoscopy (SPL) is emerging from research into clinical practice (Romanelli, 2009). SPL procedures utilize conventional as well as angled and articulating instrumentation introduced through a multilumen port (25-30 $\mathrm{mm}$ in diameter) placed, normally, in the patient navel. In addition to the clear cosmetic benefits, other possible advantages of SPL surgery compared with conventional laparoscopy include less postoperative pain, faster recovery, less adhesion formation, and reduction of convalescence time (Raman, 2007; Rané, 2008; Ponsky, 2008). However, SPL procedures are significantly hampered by limited instrument triangulation capabilities (i.e. the two surgical instruments and the endoscope are close to each other), narrow visual field through conventional laparoscopes, and both internal and external tool collisions that can considerably limit surgeon performance and jeopardize the patient's safety.
A softly-tethered camera system that can be steered from the outside of the abdomen and that is able to provide a "stadium" view, i.e. a view from above the surgical field as defined in Swain (2010), would solve most of the open issues of SPL. Additionally, if the camera size is compatible with standard laparoscopic trocars, the access used for inserting the robot can be used for a different instrument afterwards, thus avoiding a dedicated trocar for the videoendoscope.

A number of preliminary devices have been developed towards this goal. In particular, monocamera devices exploiting simple magnetic fixation and manual motion are reported in Swain (2010), Cadeddu (2009). In order to guarantee a finer control, large or complex robotic camera systems with active internal degrees of freedom have been proposed (Lehman, 2008; Hu, 2009). A small scale magnetically driven robotic camera $(12.7 \mathrm{~mm}$ in diameter, $32 \mathrm{~mm}$ in length) has already been developed with one internal degree of freedom (DoF), based on an innovative Magnetic Internal Mechanism (MIM) (Simi, 2010, 2012; Valdastri, 2010). Nevertheless, large size, poor stability, reliability and, or limited manoeuvrability or motion range, remain main drawbacks. 


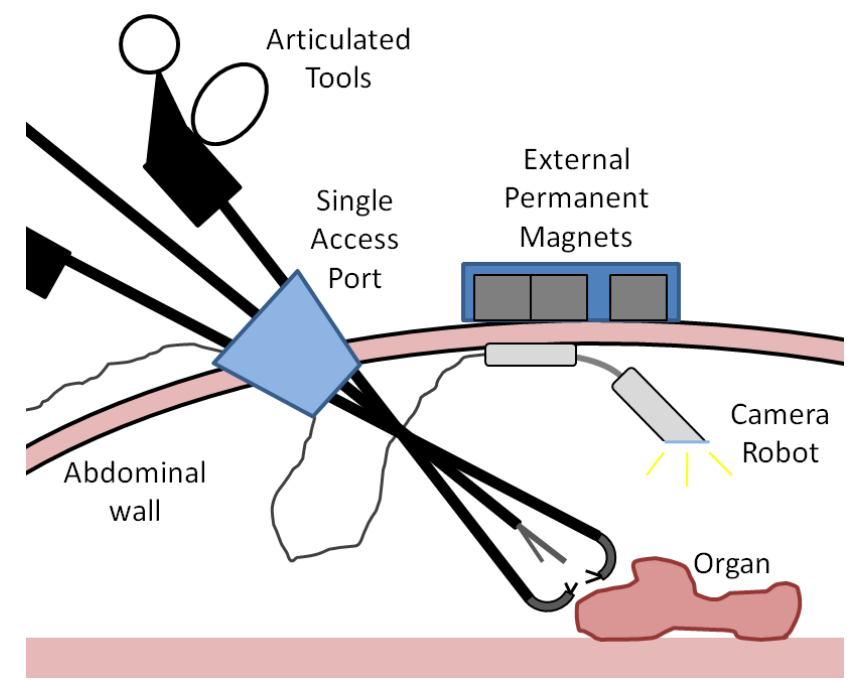

Figure 1. Schematic representation of an SPL surgical scenario using the novel camera robot based on the compliant MLS.

Compliant mechanisms offer great promise in providing new and better solutions to many mechanical design problems, thus a surgical robotic device based on these mechanisms has a number of potential advantages over traditional and current robotic systems. The monolithic nature of compliant mechanisms have the advantage of no wear debris, no pinch points, and no need for lubrication, all of which are critical in the sensitive internal body environment. In addition, single-piece production, reduces the manufacturing and assembling time and cost as well as the weight, while also the sterilization processes is simplified (Kota, 2005). Finally, from a mechanical point of view, compliant mechanisms are easily miniaturized, do not have the backlash common in rigid-body mechanisms, reduce maintenance and increase the reliability in harsh environments, such as abdominal cavity (Howell, 2001).

In this paper, we present the design, modeling and fabrication of a novel compliant Magnetic Levitation System (MLS) for a wired endoscopic surgical camera. Considering the concept known as Magnetic Anchoring and Guidance System (MAGS) (Park, 2007; Zeltser, 2007, 2008), the small device is intra-abdominally moved or anchored by External Permanent Magnets (EPMs) placed on the abdominal skin. However the main benefit stems from the robotized MLS, that exploits a static external magnetic field to provide a fine tilt motion of the camera. Furthermore, from a surgical point of view, the thin flexible cable, which guarantees powering and real time signal transmission, leaves the access port almost free, thus allowing the insertion of an additional tool (Fig. 1).

The paper is structured as follows. First of all, the overall system principle of operation is described. A nonlinear analytical static analysis is then performed for dimensioning

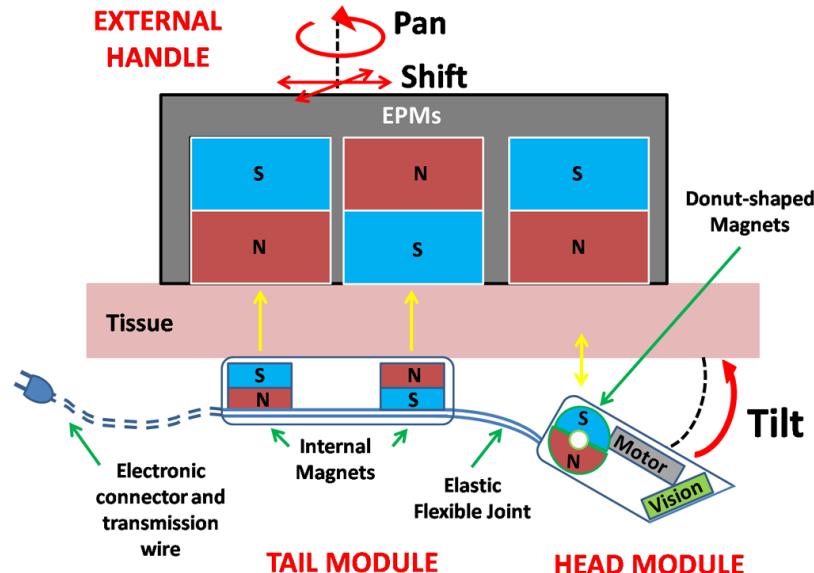

Figure 2. Schematic representation of the entire robotic endoscope with the compliant MLS. The interaction between internal and external magnets and the 4 related degrees of freedom are underlined by yellow and red arrows, respectively.

the innovative system. To this end, the compliant segment (namely the flexible joint) is modeled mathematically, and the governing equations are derived and solved analytically in a nonlinear form. The results are compared to those of finite element method (FEM). Based on the system fixed parameters and geometrical constraints, the flexible joint was designed to provide the best tilt span. Finally the robot with compliant MLS was fabricated, assembled and tested.

\section{Concept and modeling}

\subsection{Principle of operation analysis}

As represented in Fig. 2, the overall proposed surgical platform is composed by the wired robotic endoscope based on the compliant MLS and an external handle embedding 3 permanent magnets. The robotic camera is composed of two modules, the head and the tail, connected together by the flexible joint. The head embeds the vision system and a couple of donut-shaped and diametrically magnetized magnets that can be rotated by an internal brushless motor. The tail hosts two permanent magnets that, once coupled with the external ones, provide anchoring and stability to the robot. In addition, the magnetic link between external and on-board magnets allows a coarse manual manoeuvring ( $3 \mathrm{DoF})$. The concept of MLS exploits the internal magnets motorized rotation to generate an internal variable magnetic field, thus allowing for a wide-range controlled bending of the head module without any external magnets motion. Considering different magnetic actuation systems (e.g. Zeltser, 2007), the MLS guarantees a finer and more precise robotized motion, also greatly reducing the encumbrance on the patient abdomen. Small internal magnets rotation permits to obtain sufficient 

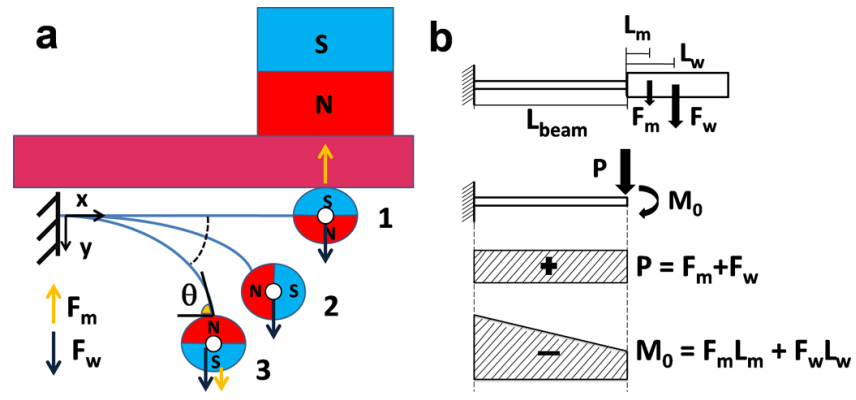

Figure 3. (a) Schematic representation of compliant MLS operation. In the equilibrium point (2) the magnetic force is negligible. (b) Force analysis and distribution considering the flexible joint like an isotropic homogeneous linear elastic beam and the head as a nondeformable body. $L_{\mathrm{w}}$ and $L_{\mathrm{m}}$ are the distances from the beam tip to the centre of gravity and the centre of donut-shaped magnets respectively. $L_{\text {beam }}$ is the elastic beam length.

forces on the head module providing a wide span tilt DOF without altering substantially the main surrounding magnetic field. Otherwise, relative motions between the external magnets (as translation or rotation) obviously create greatly variable strong magnetic fields, giving complex problem of stability, precision and controllability of the DOFs. Attractive or repulsive magnetic forces can be exerted on the robot head by the means of the complete rotation $\left(360^{\circ}\right)$ of the donutshaped magnets. In principle, this allows to achieve a $90^{\circ}$ wide tilt motion of the camera point of view, thus enabling an effective triangulation with the two surgical instruments. To effectively implement robot head levitation, an equilibrium among weight force, flexible joint stiffness and magnetic force must be guaranteed for the complete range of operation. A theoretical analysis must be performed for a proper dimensioning of the MLS and the following assumptions must be made to facilitate modeling.

The tail frame can be considered as rigidly anchored to a crushproof layer of tissue. Additionally, the flexible joint is assumed to be inextensible, rigid in shear, and of constant circular cross-section. In particular the assumption of circular cross section was made for simplicity and to define an equal out of plane stiffness. The material is assumed to follow linear elastic stress-strain behavior, homogeneous and to be isotropic. The beam is fixed at one side and subjected to two different forces (weight $F_{\mathrm{w}}$ and magnetic force $F_{\mathrm{m}}$ ) on the other end tip. Given this, three basic configurations define the behaviour of the MLS, i.e. as the donut-shaped magnets rotate, the magnetic force can have either the same (Fig. 3a3 ) or the opposite (Fig. 3a-1) orientation of the weight force, or it can be negligible (Fig. 3a-2). We refer to this last configuration as equilibrium point and it represents the static condition defined as equilibrium between weight force and beam stiffness. Correct operation of the MLS must always guarantee sufficient magnetic attraction to lift the robot head from the equilibrium point to the $0^{\circ}$ tilt position (1), whereas the repulsive magnetic force can give an extra deformation by pushing down the head from the equilibrium to the maximum tilt angle (3).

To correctly describe the flexible joint deformation behaviour, it is important to define other specific MLS features. In particular, the weight force is assumed as acting at the head module centre of gravity, while the magnetic force acts on the donut-shaped magnets and not on the flexible joint side. However, if we consider the head module as a non-deformable body, the two forces can be supposed to be applied at the connection between the flexible joint and the module, providing their respective moment in the same point (Fig. 3b). In order to maximize the camera tilt angle, the largest bending angle for an equilibrium point must be reached while the magnetic force still overcomes the head module weight force (assuming the donut-shaped magnets are oriented as in Fig. 3a-1).

Considering defined the features of the head module (Simi, 2010), the tail module and the EPMs, the only remaining free part for maximizing the camera span was the compliant joint. Equations (derived from the Euler-Bernoulli model) can be applied to describe the deformation of a linear elastic isotropic beam, providing information about the distal side displacement and the bending angle (Wang, 2008; Belendez, 2002). The mathematical model and a direct nonlinear solution (DNS) were applied to predict the best equilibrium point and to design the flexible joint.

\subsection{Compliant joint}

\subsubsection{Mathematical modeling}

The Bernoulli-Euler equation states that the bending moment is proportional to the beam curvature, which reads as follows (Gere, 1985):

$M=E I \Phi$

where $M$ is the bending moment, $\Phi$ is the curvature, $E$ is Young's modulus, $I$ is the moment of inertia of beam. $E I$ is also called the bending stiffness of the beam.

The exact expression for the curvature, $\Phi(s)$, is

$\Phi=\frac{d \theta}{d s}$

and the slope, $\theta(s)$ is defined by

$\cos (\theta)=\frac{d x}{d s}$

where $s$ is the arc-coordinate along the neutral axis of the beam from the fixed end.

For a cantilever beam under vertical end concentrated force, $P$, and end moment, $M_{0}$, as it is shown in Fig. 4, the bending moment at any coordinate of beam length is

$M(s)=P(L-a-x)+M_{0}$ 


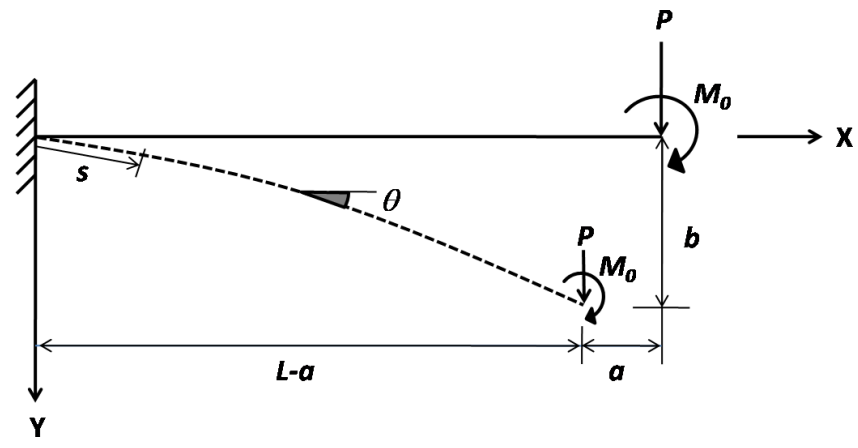

Figure 4. Flexible joint as a cantilever beam subjected to end point load and moment.

Once differentiating Eq. (4) respect to $s$ and substituting Eq. (3), the equilibrium between the internal bending moment, $M(s)$, and end point load, $P$ is found in the following form

$\frac{d M}{d s}+P \cos (\theta)=0$

Defining the dimensionless force, $\alpha$, dimensionless end moment, $\beta$, and dimensionless length, $\xi$, as follows:

$\alpha=\frac{P L^{2}}{E I}, \quad \beta=\frac{M_{0} L}{E I} \quad$ and $\quad \xi=\frac{s}{L}$

and substituting the moment curvature relation Eq. (1) and the curvature relation from Eq. (2) into equilibrium equation Eq. (5) results in the following governing equation:

$\frac{d^{2} \theta}{d \xi^{2}}+\alpha \cos (\theta)=0$

where the boundary conditions are

$$
\left.\theta\right|_{\xi=0}=0 \quad \text { and }\left.\quad \frac{d \theta}{d s}\right|_{\xi=1}=\beta
$$

\subsubsection{Direct nonlinear solution}

Since the flexible member undergoes large deflection, linearized beam equations are no longer valid. Nonlinear equations must be used that account for the geometric nonlinearities caused by large deflections. In fact, if the deflections are large, the flexible cantilever beam with constant cross section and linear material properties may be out of the range of linearized beam deflection equations, and elliptic integral solutions or non linear finite element analysis are used to perform the analysis. Large deflection elliptic-integral equations show that for a flexible cantilever beam with a force at the free end, the free end follows a nearly circular path, with some radius of curvature along the beam's length.

The DNS (Morsch, 2009) for the large deflection analysis of an initially curved cantilever beam under end point loads (Fig. 4) was selected. Homotopy perturbation method
(HPM) (He, 1999) is used as to obtain the semi-exact solution of rotation angle of any coordinate of beam length and the corresponding trajectory position, after a simple integration in a close form parametric expression (Morsch, 2009). For verification, the results obtained from this section are compared to those of FEM as will be presented and discussed in Sect. 4.

To solve the high order nonlinear Eq. (7), without the linear assumption of $\cos (\theta)=1$, the Taylor series expansion of $\cos (\theta)$ has been considered and the HPM (Morsch, 2009) has been applied. Applying the HPM to Eq. (7), we construct a homotopy in the following form:

$H(\theta ; p)=(1-p)\left[\frac{d^{2} \theta}{d \xi^{2}}\right]+p\left\{\frac{d^{2} \theta}{d \xi^{2}}+\alpha\left[1-\frac{\theta^{2}}{2 !}+\frac{\theta^{4}}{4 !}\right]\right\}$

where $p$ is representing homotopy parameter. According to the homotopy-perturbation method, we assume that the solution of Eq. (7) can be expressed in a series of $p$ as below:

$\theta(\xi ; p)=\theta_{0}(\xi)+\sum_{m=1}^{\infty} \theta_{m}(\xi) p^{m}$

Substituting $\theta$ from Eq. (10) into Eq. (9) and rearranging based on power series of $p$, we have an equation system including $m+1$ equation to be simultaneously solved; $m$ is the order of $p$ in Eq. (10). For an accurate result (error not greater that $5 \%$ ) and at the same time minimizing the computational time, $m=2$ has been considered (Tolou, 2012) as follows:

$p^{0}: \frac{d^{2} \theta_{0}}{d \xi^{2}}=0$

$p^{1}: p\left\{\frac{d^{2} \theta_{1}}{d \xi^{2}}+\alpha\left[1-0.500 \beta^{2} \xi^{2}+0.417 e-1 \beta^{4} \xi^{4}\right]\right\}=0$

$p^{2}: p\left\{\frac{d^{2} \theta_{2}}{d \xi^{2}}+\alpha^{2}\left[\begin{array}{l}\beta^{7}\left(0.139 e-2 \xi^{4}-0.232 e-3 \xi^{9}\right) \\ +\beta^{5}\left(0.835 e-2 \xi^{7}-0.279 e\right. \\ \left.-1 \xi^{4}-0.834 e-2 \xi^{2}\right) \\ +\beta^{3}\left(0.167 \xi^{2}+0.167 \xi^{4}\right. \\ \left.-0.125 \xi^{5}\right)+\beta\left(0.500 \xi^{3}-\xi^{2}\right)\end{array}\right]\right\}=0(11 \mathrm{c})$

Solving system of Eq. (11) subsequently with the effective boundary conditions results in $\theta$ 's

$\theta_{0}(s)=\beta \xi$

$$
\begin{gathered}
\theta_{1}(\xi)=\alpha \xi\left(-0.139 e-2 \beta^{4} \xi^{5}+0.417 e-1 \beta^{2} \xi^{3}\right. \\
\left.-0.500 \xi+0.834 e-2 \beta^{4}-0.167 \beta^{2}+1\right) \\
\theta_{2}(\xi)=\alpha^{2} \xi\left[\begin{array}{l}
\beta^{7}\left(0.211 e-5 \xi^{10}-0.463 e-4 \xi^{5}+0.255 e\right. \\
-3)+\beta^{5}\left(-0.116 e-3 \xi^{8}+0.695 e-3 \xi^{3}\right. \\
\left.+0.930 e-3 \xi^{5}-0.732 e-2\right)+\beta^{3}(0.298 e \\
\left.-2 \xi^{6}-0.557 e-2 \xi^{5}-0.139 \xi^{3}+0.682 e-1\right) \\
+\beta\left(0.833 e-1 \xi^{3}-0.250 e-1 \xi^{4}-0.208\right)
\end{array}\right]
\end{gathered}
$$


and the final result is built up with the $\theta$ 's, using the following equation

$\theta_{\text {final }}=\lim _{p \rightarrow 1}\left[\theta_{0}+\sum_{m=1}^{+\infty} p^{m} \theta_{m}\right]$

An increase in $m$ improves the accuracy in Eq. (10) with $\theta$ obtained from Eq. (13), the non-dimensional trajectory can be calculated directly using the following equations (Morsch, 2009):

$$
\begin{aligned}
& u_{y}(\xi)=\int_{0}^{\xi} \sin (\theta) d \xi \\
& u_{x}(\xi)=\int_{0}^{\xi} \cos (\theta) d \xi
\end{aligned}
$$

\subsubsection{FEM evaluation}

In order to provide a basis for evaluation of the accuracy of presented approach, finite element modeling (FEM) of the discussed problem was performed. The stresses, strains and displacements were analyzed using a commercially available FEM package, ANSYS $^{\text {TM }} 11.0$ (ANSYS Inc. Manual, 2008). Because of large deflections, a non-linear static analysis has been performed. All the 1000 elements were created using the BEAM 188 element. This uni-axial element gives the shortest computation time while the actual 3-D properties can also be provided using the beam section defining capability of ANSYS. This two node element has finite strain and has six degrees of freedom at each node with tension, compression, and bending capabilities. The material is assumed to follow linear elastic stress-strain behavior and to be isotropic. The beam is fully clamped at one end and end point load and moment has been applied on free end node.

\section{Dimensional design and fabrication}

\subsection{External permanent magnets}

Because the EPMs must be easily handled by the medical doctors, three off-the-shelf (KJ Magnetics, Jamison US), cubic $(25.5 \mathrm{~mm} \times 25.5 \mathrm{~mm} \times 25.5 \mathrm{~mm})$ magnets $(\mathrm{NdFeB}, \mathrm{N} 52)$ were selected as the best compromise between high magnetic field and size to generate the external magnetic field both for the MAGS and the MLS.

\subsection{Head module}

Considering the endoscopic camera robot described in Simi (2010), a very similar prototype was fabricated as head module of the new robot. An improvement regarded orientation of the camera view direction assembled with an angle of $10^{\circ}$. The head module (Fig. 5) consists of a small and

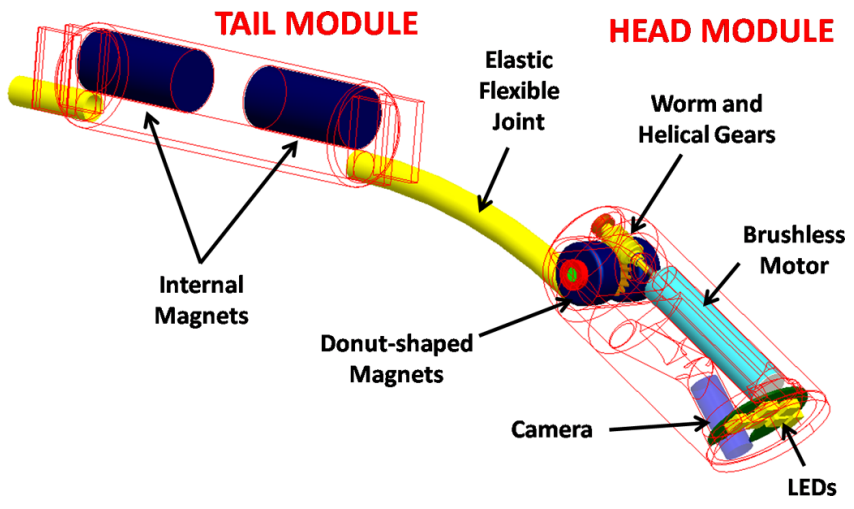

Figure 5. Threedimensional model of the designed prototype. The head module embeds ring magnets, a motor with gears and a vision system; the tail module embeds only two magnets for the system anchoring and external rough motion. The device results $12.7 \mathrm{~mm}$ in diameter and $95 \mathrm{~mm}$ in length.

light plastic cylinder ( $12.7 \mathrm{~mm}$ in diameter, $34 \mathrm{~mm}$ in length and $7.5 \mathrm{~g}$ in weight, fabricated by rapid prototyping) that embeds two donut-shaped magnets $(6.3 \mathrm{~mm}$ and $1.6 \mathrm{~mm}$ in external and internal diameter respectively and $3.2 \mathrm{~mm}$ in thickness) diametrically magnetized ( $\mathrm{NdFeB}, \mathrm{N} 52)$ and linked by means of two gears (worm and helical gears) to a brushless motor (SBL04-0829-PG337 Namiki Precision Jewel Co. Ltd., Tokyo, Japan). It also includes a vision system composed of a thin CCD camera (MO-B802-105 Misumi Electronics Corp., Taiwan) and a set of high efficiency white LEDs (NESW007BT, Nichia Corp., Tokushima, Japan) for illumination. An external push-button console was used for activating the internal motor and defining the rotation of the donut-shaped magnets.

\subsection{Tail module}

The tail module is $11 \mathrm{~mm}$ in diameter, $40 \mathrm{~mm}$ in length and $10.5 \mathrm{~g}$ in weight (see Fig. 5). Given the geometry and the properties of the EPMs, considering the average human abdominal wall thickness as $30 \mathrm{~mm}$ (Song, 2006) and in order to satisfy the MAGS main technical requirements (Cahill, 2009), two commercially available (KJ Magnetics, Jamison US) cylindrical $(6.35 \mathrm{~mm}$ in diameter and $12.7 \mathrm{~mm}$ in length) magnets $(\mathrm{NdFeB}, \mathrm{N} 52)$ were embedded in the tail. A long longitudinal hole in the frame body allowed the wires to run through the module.

\subsection{Flexible joint}

The flexible joint is the last part to dimension in order to obtain optimal MLS functionality, guaranteeing a sufficient magnetic force to lift the head from the equilibrium point while also maximizing the camera tilt span angle.

In order to keep fabrication and assembling of the entire camera easy and due to the need for wired connections 


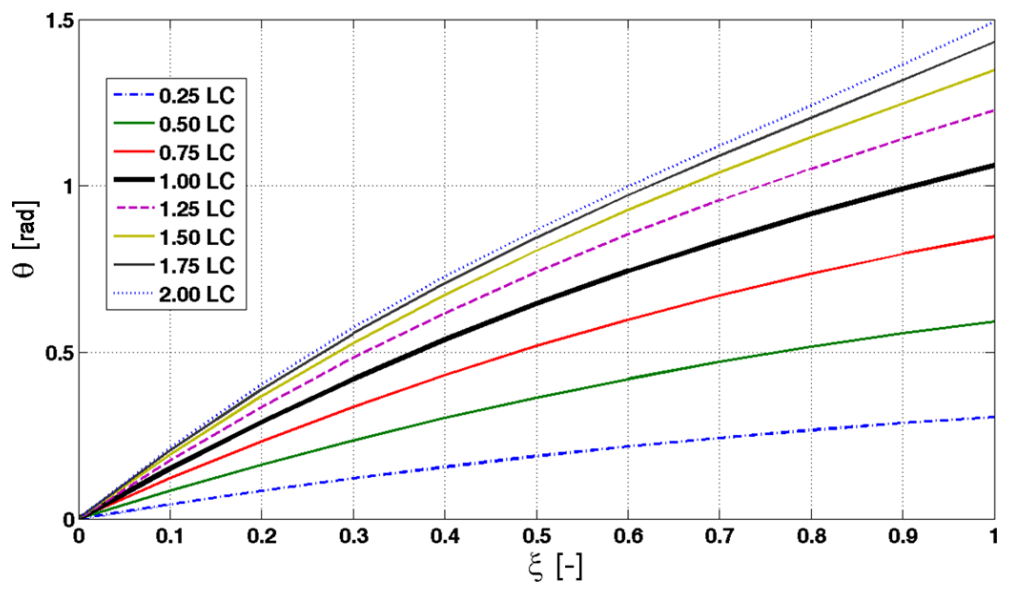

Figure 6. Slope along beam length of the flexible joint subject to different load cases (LC) from presented method. LC: $\beta=0.663$ [ - ], $\alpha=1.137[-]$ corresponds to $F_{\mathrm{w}}=73.5 \mathrm{mN} ; L_{\mathrm{w}}=14 \mathrm{~mm} ; I=2.5 \times 10^{-13} \mathrm{~mm}^{4} ; E=150 \mathrm{MPa}$.

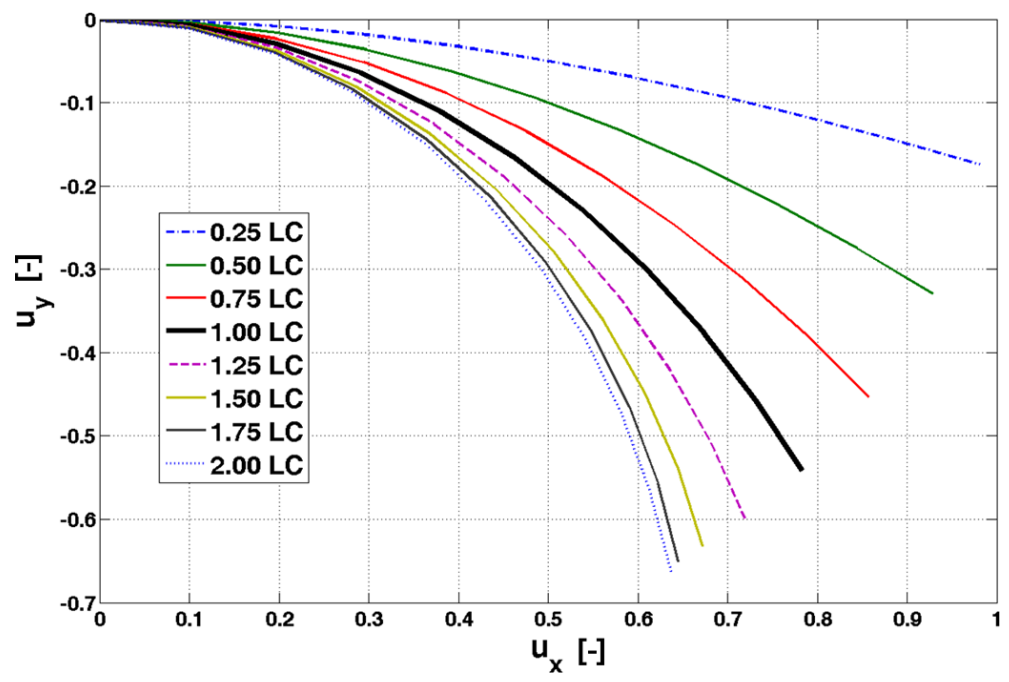

Figure 7. Trajectory position of the flexible joint subject to different load cases (LC) from presented method. LC: $\beta=0.663$ [-], $\alpha=1.137$ [-] corresponds to $F_{\mathrm{w}}=73.5 \mathrm{mN} ; L_{\mathrm{w}}=14 \mathrm{~mm} ; I=2.5 \times 10^{-13} \mathrm{~mm}^{4} ; E=150 \mathrm{MPa}$.

between the two robotic modules (tail and head) for power supply and data transmission, an elastic polytetrafluoroethylene (PTFE) tubular sheath (1.5 mm in diameter) completely filled with 9 electric wires was used directly as flexible joint. A circular section was defined and the flexible joint composite material Young's modulus $(E)$ was then experimentally measured equal to $150 \mathrm{MPa}$ assuming negligible hysteresis.

Given the geometry, weight and magnetic content of the head module, the external magnets and also the joint, we relate the compliant MLS behaviour to different joint length. Due to the magnetic force between external magnet and donut-shaped magnets changes with the beam free tip displacement as function of joint deflection and length, only the equilibrium condition was analyzed and satisfied, then the maximum angle span was evaluated.
Based on the known parameters $\left(F_{\mathrm{w}}=73.5 \mathrm{mN} ; L_{\mathrm{w}}=\right.$ $\left.14 \mathrm{~mm} ; \quad I=2.5 \times 10^{-13} \mathrm{~mm}^{4} ; \quad E=150 \mathrm{MPa}\right)$ namely load case (LC), and the DNS, the trend lines of the beam equilibrium point (magnetic force neglected), in terms of nondimensional deflection of the beam, the relative bending angle and the displacement of the free tip were obtained (Figs. 6 and 7). Considering the load that acts on the beam free tip as a composition of force and moment defined only by the head features, in our case (LC), the obtained plots describe also the compliant joint behaviour under a fraction of the LC.

After the beam deflection analysis some discrete FEM with COMSOL Multiphysics 3.4 (COMSOL Inc., Sweden) were used to evaluate the magnetic field trend and especially the vertical magnetic attraction force between the donutshaped magnets and the external magnet. Considering Fig. 7 , the two donut-shaped magnets were positioned as a function 




Figure 8. FEM analysis of the magnetic interaction between donutshaped magnets and the external magnet in order to evaluate magnetic force components $\left(F_{\mathrm{m}}\right)$ for different equilibrium points. All the internal and external magnets were considered. The mesh consisted of about 1350000 elements with a minimum quality ratio of 0.3 . In this screenshot is showed the magnetic attraction force evaluation in the equilibrium point for a defined beam length of $24 \mathrm{~mm}$.

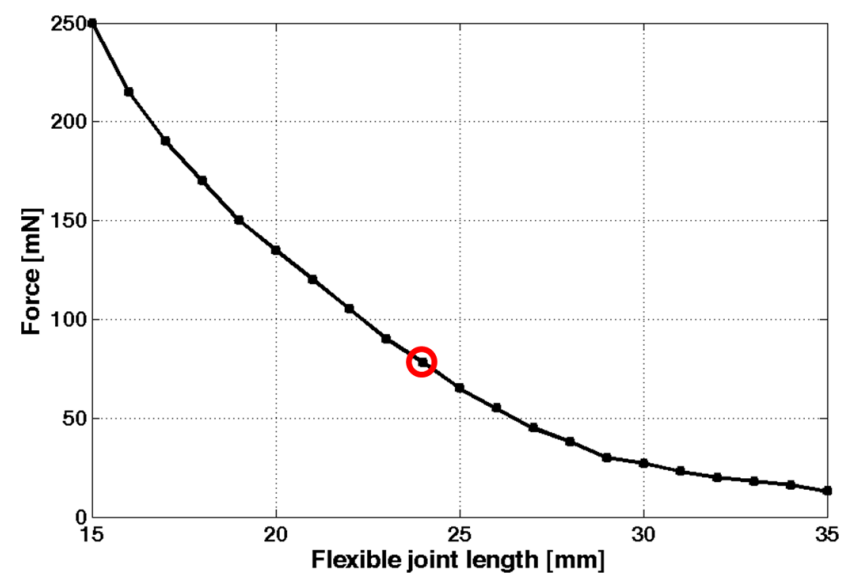

Figure 9. Trend line of the vertical magnetic attraction force between the MLS external and donut-shaped magnets in equilibrium point (Fig. 2a-2). The dots represent the discrete FEM analysis results. The red circle highlights the $F_{\mathrm{m}}$ equal to $F_{\mathrm{w}}$ for a $24 \mathrm{~mm}$ $L_{\text {beam. }}$.

of the specific beam tip equilibrium point (but assumed oriented as in Fig. 3a-1) related to beam length in a range between $15 \mathrm{~mm}$ and $35 \mathrm{~mm}$ (with a $L_{\text {beam }}$ step of $1 \mathrm{~mm}$ ) (Fig. 8). The magnetic attraction force trend line was then represented in Fig. 9.

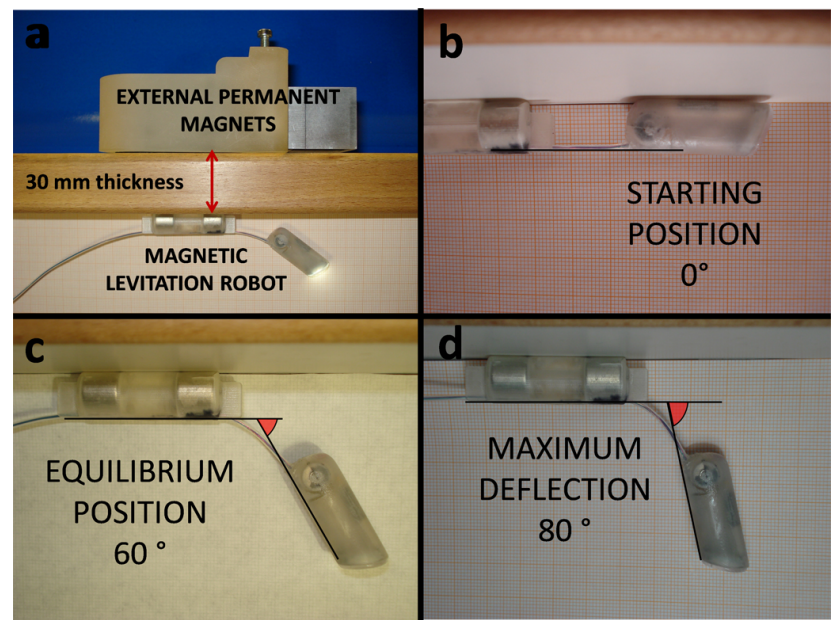

Figure 10. Evaluation of the tilt span. (a) Bench test scenario. (b) The maximum attraction force keeps the robot head near the abdominal wall. (c) Equilibrium point: $F_{\mathrm{m}}$ is negligible and the head tilt angle is $60^{\circ}$. (d) The repulsive magnetic force pushes down the head that reaches a final angle of $80^{\circ}$.

\section{Results and discussion}

Based on the collected data (Fig. 9) a beam length of $24 \mathrm{~mm}$ results in the highest bending angle that still guarantees a magnetic force $\left(F_{\mathrm{m}}=77 \mathrm{mN}\right)$ sufficient to lift the head module from the equilibrium point to the $0^{\circ}$ tilt configuration (Fig. 3a-1). Considering Fig. 6, an angle of $60^{\circ}$ (1.05 rad) was reached in the equilibrium point due to the weight force of the head and the elastic joint properties.

Once the flexible joint was modeled and dimensioned, it was assembled with the other two modules (head and tail) and the MLS was tested. The tilt angles were measured through lateral views of the robot placed against a millimeterscaled background. Without external magnet (absence of magnetic force) the head reaches the equilibrium point with an angle of $60^{\circ}$ in relation to the horizontal wall, as predicted. Replacing the external magnet on the wall and rotating the donut-shaped magnets, the robot head can levitate with a total smooth and fine tilt span ranging from $0^{\circ}$ up to $80^{\circ}$ as showed in Fig. 10. During the span between $0-60^{\circ}$ the magnetic force compensates the weight force until the equilibrium point, whereas from 60 to $80^{\circ}$ the repulsive magnetic force pushes down the head.

The experimental results are fully comparable with model predictions. The entire design procedure is consistent, correct and reliable to easily dimension a compliant MLS. Thanks to the model presented in this paper, further adjustments of system performances can be easily implemented in future prototypes or used to develop similar devices.

Regarding the mathematical flexible joint deflection analysis, the DNS not only overcomes the inaccuracy problem of a linear solution, also eliminates some shortcomings in 
Table 1. Comparison of dimensionless deflections, $x[-]$ and $y[-]$, and slope, $\theta$ [rad], of free end tip from FEM and DNS as the absolute values and relative errors* for the case study; load case (LC) $=\beta=0.663[-], \alpha=1.137[-]$ corresponds to $F_{\mathrm{w}}=73.5 \mathrm{mN} ; L_{\mathrm{w}}=14 \mathrm{~mm}$; $I=2.5 \times 10^{-13} \mathrm{~mm}^{4} ; E=150 \mathrm{MPa}$.

\begin{tabular}{|c|c|c|c|c|c|c|c|c|c|}
\hline \multirow[t]{2}{*}{ Load } & \multicolumn{3}{|c|}{ FEM } & \multicolumn{3}{|c|}{ DNS } & \multicolumn{3}{|c|}{ Error [\%] } \\
\hline & $x$ & $y$ & $\theta$ & $x$ & $y$ & $\theta$ & $x$ & $y$ & $\theta$ \\
\hline $0.25 \mathrm{LC}$ & 0.019 & 0.174 & 0.305 & 0.019 & 0.174 & 0.305 & 1.302 & 0.130 & 0.144 \\
\hline $0.5 \mathrm{LC}$ & 0.071 & 0.328 & 0.588 & 0.072 & 0.329 & 0.593 & 2.126 & 0.381 & 0.811 \\
\hline $0.75 \mathrm{LC}$ & 0.140 & 0.451 & 0.840 & 0.143 & 0.453 & 0.848 & 2.124 & 0.485 & 0.956 \\
\hline $1 \mathrm{LC}$ & 0.216 & 0.544 & 1.059 & 0.217 & 0.541 & 1.061 & 0.585 & 0.577 & 0.217 \\
\hline $1.25 \mathrm{LC}$ & 0.290 & 0.613 & 1.248 & 0.281 & 0.598 & 1.226 & 3.215 & 2.448 & 1.784 \\
\hline $1.5 \mathrm{LC}$ & 0.360 & 0.663 & 1.414 & 0.328 & 0.632 & 1.348 & 8.976 & 4.706 & 4.651 \\
\hline $1.75 \mathrm{LC}$ & 0.425 & 0.700 & 1.560 & 0.356 & 0.651 & 1.431 & 16.145 & 6.940 & 8.256 \\
\hline $2 \mathrm{LC}$ & 0.483 & 0.726 & 1.690 & 0.363 & 0.662 & 1.493 & 24.811 & 8.773 & 11.664 \\
\hline
\end{tabular}

* FEM results have been the references values.

conventional numerical methods like elliptic integrals, such as implementation difficulties and accuracy problems due to table look-up. Residuals from the presented method are significantly less than from the linear solution. Practically, they can be considered negligible. This gives us a reason to claim that the presented method is remarkably accurate. We have successfully utilized DNS to handle the geometric nonlinearity caused by large deflection of the cantilever beam. It is evident that the method is very powerful and efficient for solving this kind of problems arising in compliant mechanisms and other mechanical systems, and presents a rapid convergence for the solutions. The parametric solution from Eqs. (12), (14) (with parameters of loads and coordinate along the neutral axis of the beam) can give rise to physical understanding the problem and better optimization performance in terms of computational time and minimizing objective functions. It was found that the maximum inaccuracy for proposed solutions occurs at the free end point and amounts to less than $1 \%$ as compared to FEM (Table 1). However, for higher loads than the design load case, inaccuracy increase which can be compensated by increasing the number of iterations in Eq. (10). As shown in this table, the trajectory, $x$ and $y$, are mainly of higher inaccuracy relative to slope which is due to Taylor series assumption for the first three terms of the sin and cos functions in Eq. (14). This also brings some irregular trends in accuracy for $y$ from high nonlinearity of the parameters during the manipulation. The slope of any coordinates along the neutral axis of the beam and the trajectory of the free end tip for load cases specified in Table 1 are shown in Figs. 6 and 7, respectively. As shown in these figures, with an increase in the load case, the deflection becomes larger and more nonlinear. The larger nonlinearity of trajectory (Fig. 7) compared to slope (Fig. 6) can be explained mathematically from Eq. (14) due to nonlinear sin and cos functions. The von Misses stress from FEM has found 11.7 MPa for the maximum LC considered in Table 1, Figs. 6 and 7 which was far below the yield stress.

\section{Conclusions}

The compliant MLS represents a novel concept to obtain an internal DoF for a robotic camera device immersed in a magnetic field. A nonlinear mathematical modeling and parametric direct nonlinear solution were successfully presented to predict the trajectory of compliant joint for different load cases. This leads to a successful parametric design for a successful and straightforward dimensioning and may be applied to similar or more complex problems. Based on the modeling we designed and fabricated a MLS working prototype for an endoscopic camera. It is $12.7 \mathrm{~mm}$ in diameter, $95 \mathrm{~mm}$ in length and $18 \mathrm{~g}$ in weight. It is composed of two robotic modules (tail and head) linked by an elastic flexible joint. Under the set of conditions, detailed above, when the embedded motor is activated, the robot head module can levitate with a span ranging from 0 to $80^{\circ}$, thus guaranteeing a high motion of the point of view $\left(10-90^{\circ}\right)$. Finally, use such MLS permits to actuate a DOF having all the benefits of a compliant joint as discussed in the introduction.

The small robotic endoscope can be inserted through a conventional trocar and fixed on the abdominal wall. Space in the access port is only taken by thin cables $(1.5 \mathrm{~mm})$, thus leaving free space for the access of additional instrumentation. A rough position along the abdominal wall can be achieved by dragging the robot with the motion of the EPMs. Once positioned, a fine tilt orientation of the camera can be obtained by exploiting only the MLS. Additionally, since the tilting motion is not manual as in Cadeddu (2009), but motorized, image stability and motion resolution can be greatly enhanced. Accordingly, the novel robotic endoscope has the potential to restore triangulation for the surgeon and to reduce both instrument collision and procedure invasiveness. In laparoscopy, use of this innovative approach would reduce the number of external incisions, since a devoted access for the camera would no longer be required. Considering an SPL scenario, all the ports can be used to insert instruments, 
thus facilitating the tasks for the surgeon and also eliminating the potential conflict with the endoscopist. Some recent in vivo tests (Simi, 2011) demonstrate that tools motion (in particular insertion and retrieve), exerting sometimes relevant forces, can cause abdominal wall deformation, generating head vibration. Deflections and vibrations outside xy-plane also occurred, especially when the robot is not anchored parallelly to the floor. In this case, a gravity force component deflects the camera head out of the xy-plane. Concerning patient's breathing, no vibration were observed. Abdomen wall upon insufflations results stiff and not deformable reducing markedly breathing motions.

Further improvement will be performed to completely enhance system performance. In order to reduce head vibrations but also improve control of the next prototype a dedicated eigenfrequency study of the beam will be performed in the future also choosing new materials and section shapes.

A better and complete analysis of magnetic interaction forces (attraction-repulsion) along the free tip trend line, during joint deflection, will be evaluated as function of length. A dedicated control algorithm will be implemented to precisely relate the donut-shaped magnets rotation to the head-camera tilt angle. In fact, optimizing modeling and prototype design but also embedding sensors, we could finally implement position control or auto-adjustment for vibration compensation to achieve better stability for the entire system.

Acknowledgements. The authors would like to thank Nicodemo Funaro and all the staff of the BioRobotics Institute mechanical workshop for manufacturing the robot prototypes. This research is part of European Commission in the framework of the ARAKNES European Project EU/IST-2008-224565 and in part supported by VIDI Innovational Research Incentives Scheme grant for the project "Statically balanced compliant mechanisms", NWO-STW 7583.

Edited by: C. Kim

Reviewed by: two anonymous referees

\section{References}

ANSYS Inc.: Manual of ANSYS 11, ANSYS Inc., 2008.

Belendez, T., Neipp, C., and Belendez, A.: Large and small deflections of a cantilever beam, Eur. J. Phys., 23, 371-979, 2002.

Cadeddu, J., Fernandez, R., Desai, M., Bergs, R., Tracy, C., Tang, S., Rao, P., and Scott, D.: Novel magnetically guided intra-abdominal camera to facilitate laparoendoscopic singlesite surgery: initial human experience, Surgical Endoscopy, 23, 1894-1899, 2009.

Cahill, R. A., Lewin, R. P., Mortensen, N. J., and Jones, H.: The principles and pratice of magnetic instrumentation for natural orifice transluminal endoscopic surgery and other limited access surgical operation, Proc. IMechE, J. Mech. Eng. Sci., 224C, 1455-1461, 2009.

Gere, J. M. and Timoshenko, S. P.: Mechanics of Materials, PWS Engineering Publishers, Boston, 1985.
He, J. H.: Homotopy perturbation technique, Comput. Methods Appl. Mech. Eng., 178, 257-262, 1999.

Howell, L.: Compliant mechanics, Wiley \& Sons, New York, 2001.

$\mathrm{Hu}$, Y., Allen, P., Hongle, N., and Fowler, D.: Insertable surgical imaging device with pan, tilt, zoom, and lighting, Journal of Robotic Research, 28, 1373-1386, 2009.

Kota, S., Lu, K., Kreiner, Z., Trease, B., Arenas, J., and Geiger, J.: Design and application of compliant mechanism for surgical tools, J. Biomech. Eng.-T. ASME, 127, 981-989, 2005.

Lehman, A., Berg, K., Dumpoert, J., Wood, N., Visty, A., Rentschler, M., Platt, S., Farritor, S., and Oleynikov, D.: Surgery with cooperative robots, Comput. Aided Surg., 13, 95-105, 2008.

Loring, S., Brown, R., Gouldstone, A., and Butler, J.: Lubrication regimes in mesothelial sliding, J. Biomech., 38, 2390-2396, 2005.

Morsch, F., Tolou, N., and Herder, J. L.: Comparison of Methods for Large Deflection Analysis of a Cantilever Beam Under Free End Point Load Cases, in: Proceeding of ASME 2009 International Design Engineering Technical Conferences and Computers and Information in Engineering Conference, 30 August2 September, San Diego, CA, USA, Vol. 7, 33rd Mechanisms and Robotics Conference, Parts A and B, Paper no. DETC200986754, 183-191, doi:10.1115/DETC2009-86754, 2009.

Park, S., Bergs, R. A., Eberhart, R., Baker, L., Fernandez, R., and Cadeddu, J.: Trocar-less instrumentation for laparoscopy: magnetic positioning of intra-abdominal camera and retractor, Ann. Surg., 245, 379-384, 2007.

Ponsky, L. E., Cherullo, E. E., Sawyer, M., and Hartke, D.: Single access site laparoscopic radical nephrectomy: initial clinical experience, J. Endourol., 22, 663-666, 2008.

Raman, J. D., Bensalah, K., Bagrodia, A., Stern, J., and Cadeddu, J.: Laboratory and clinical development of single keyhole umbilical nephrectomy, Urology, 70, 1039-1042, 2007.

Rané, A. and Rao, P.: Single-port-access nephrectomy and other laparoscopic urologic procedures using a novel laparoscopic port (R-port), Urology, 72, 260-263, 2008.

Romanelli, J. R. and Earle, D. B.: Single-port laparoscopic surgery: an overview, Surgical Endoscopy, 23, 1419-1427, 2009.

Simi, M., Ciuti, G., Tognarelli, S., Valdastri, P., Menciassi, A., and Dario, P.: Magnetic link design for a robotic laparoscopic camera, J. Appl. Phys., 107, 09B302, 1-3, 2010.

Simi, M., Valdastri, P., Di Lorenzo, N., Basili, G., Menciassi, A., and Dario, P.: Preliminary in-vivo Tests with a Magnetic Levitation Camera Robot for Laparoscopic Surgery, in: Proc. of Hamlyn Symposium on Medical Robotics, London, 25-26, 19-20 June 2011.

Simi, M., Silvestri, M., Cavallotti, C., Vatteroni, M., Valdastri, P., Menciassi, A., and Dario, P.: Magnetically Activated Stereoscopic Vision System for Laparoendoscopic Single Site Surgery, IEEE/ASME Transactions on Mechatronics, in press, 2012.

Song, C., Alijani, A., Frank, T., Hanna, G., and Cuschieri, A.: Mechanical properties of the human abdominal wall measured in vivo during insufflation for laparoscopic surgery, Surgical Endoscopy, 20, 987-990, 2006.

Swain, P., Austin, R., Bally, K., and Trusty, R.: Development and testing of a tethered, independent camera for notes and sigle-site laparoscopic procedures, Surgical Endoscopy, 24, 2013-2021, 2010.

Tolou, N., Khiat, A., Zhang, G. Q., and Herder, J. L.: 
Analytical Method for Determination of Young's Modulus of Large Deflection Carbon Nanotube, Int. J. Nonlin. Sci. Num., doi:10.1515/IJNSNS.2011.300, in press, 2012.

Valdastri, P., Quaglia, C., Buselli, E., Arezzo, A., Di Lorenzo, N., Morino, M., Menciassi, A., and Dario, P.: A magnetic internal mechanism for precise orientation of the camera in wireless endoluminal applications, Endoscopy, 42, 481-486, 2010.

Wang, J., Chena, J., and Liaob, S.: An explicit solution of the large deformation of a cantilever beam under point load at the free tip, J. Comput. Appl. Math., 212, 320-330, 2008.
Zeltser, I. S. and Cadeddu, J. A.: A novel magnetic anchoring and guidance system to facilitate single trocar laparoscopic nephrectomy, Curr. Urol. Rep., 9, 62-64, 2008.

Zeltser, I. S., Bergs, R., Fernandez, R., Baker, L., Eberhart, R., and Cadeddu, J.: Single trocar laparoscopic nephrectomy using magnetic anchoring and guidance system in the porcine model, J. Urol., 178, 288-291, 2007. 